S. Kwok, M. Dopita, and R. Sutherland, eds.

\title{
Three-Dimensional Monte Carlo Simulations of Ionised Nebulae
}

\author{
Barbara Ercolano ${ }^{1}$, Christopher Morisset ${ }^{2}$, Michael J. Barlow ${ }^{1}$, Xiao-wei \\ $\mathrm{Liu}^{1}$, Peter J. Storey ${ }^{1}$ \\ ${ }^{1}$ Department of Physics and Astronomy, University College London, \\ Gower Street, London, WC1E 6BT,UK \\ ${ }^{2}$ Institut d'Astrophysique de Marseille, France
}

\begin{abstract}
A three-dimensional self-consistent photo-ionisation code is developed using Monte Carlo techniques in order to build realistic models of photoionised nebulae of arbitrary geometry and density distribution. The code's results are benchmarked against those of estabilished codes. Two models are computed for the planetary nebula NGC 3918 using two different density distributions. The results of the two models are compared with the observations.
\end{abstract}

\section{Introduction}

Observational evidence indicates the non sphericity of almost every type of astronomical nebula. In particular, this is true for planetary nebulae (PNe). Realistic photoionization models of such objects require a two- or three-dimensional treatment where the stellar and diffuse field radiative transport is solved properly. The Mocassin code is a self-consistent 3D code which uses Monte Carlo techniques to handle the radiative transport problem for arbitrary geometry and density distributions. This paper describes benchmark results of the code and application to the PN NGC 3918.

Mocassin was tested against some of the benchmark models discussed at the Workshop on Ionised Plasma codes held at Lexington in November 2000 (Ercolano 2002; Péquignot et al. 2002). The results obtained for the Meudon/ Lexington benchmark optically thick homogeneous planetary nebula (PN150) were compared with those obtained by other established codes. The comparison shows that Mocassin's results are in satisfactory agreement with those of the classical codes (Ercolano, 2002).

\section{The modelling of NGC 3918}

NGC 3918 is a well known and widely studied PN. Clegg et al. (1987) constructed a photoionisation model of this object using a biconical "pole-on" geometry. The model was used to derive the physical parameters of the central star and the nebular chemical abundances. Corradi et al. (1999) analyzed optical images and high resolution slit spectra of NGC 3918. Based on the evolution- 
ary models of Mellema (1993) they assumed a geometry consisting of a bright elliptical inner shell from which two fainter protrusions extend in the long axis direction.

We have constructed two photoionisation models (A\&B) for NGC 3918. In model A, the density distribution assumed by Clegg et al. (1987) was adopted. In model $\mathrm{B}$ a density distribution similar to that proposed by Corradi et al. (1999) was used. The stellar parameters and abundances used for both models are those derived by Clegg et al. (1987). The emission spectra predicted by the two models were compared to Clegg et al.'s model and to the observed values. Mocassin calculates the luminosity in the emission lines at each grid cell. Model A produces a spectrum which is in general agreement with the model predictions of Clegg et al. and with the observed values. Most discrepancies between the current work and Clegg et al.'s can be attributed to the different atomic data sets used. The output spectrum from model B does not reproduce the observed values. The low ionization species are too weak while the high excitation lines are too strong compared to observations. More work is needed on model B based on Corradi et al.'s work in order to investigate whether better agreement with the observed spectrum can be reached by adjusting the density distribution parameters.

Using IDL routines, the nebula can be turned by any angle and projected images in any line can be produced. The intensity corresponding to a given aperture can be calculated and compared with the observations. Line profiles can also be computed by assuming a velocity field. The HeII 4686 line profiles were calculated for the two models by assuming different velocity fields. Model $A$ uses a radial velocity field, while model $B$ uses the bipolar outflow described in the Corradi et al. (1999) paper. The HeII 4686 line profiles at several points on the nebula and position-velocity (PV) diagrams and isovelocity contours for models $\mathrm{A}$ and $\mathrm{B}$ were also produced. The tables and figures describing the above results were presented in the poster for this conference and will be included in Ercolano et al. (2002). The projected maps and the line profiles obtained are those one would expect from the geometries assumed in the two models. These, combined with the PV diagrams, can prove a powerful tool when observations are available for comparison.

\section{References}

Clegg, R. E. S., Harrington, J. P., Barlow, M. J., Walsh, J. R. 1987, ApJ, 314, 551

Corradi, R. L. M., Perinotto, M., \& Villaver, E., Mampaso, A., Gonçalves, D. R. 1999, ApJ, 523, 721

Ercolano, B. 2002, in ASP Conf. Ser., in press

Mellema, G. 1993, Ph.D. thesis, Univ. Leiden

Pequignot, D., Ferland, G., Netzer, H., Kallman, T., Ballantyne, D. R., Dumont, A.-M., Ercolano, B., Harrington, P., Kraemer, S., Morisset, C., Nayakshin, S., Rubin, R. H., Sutherland, R. 2002, in ASP Conf. Ser., in press 